\title{
Immunohistochemical characterization of the chick marginal retina
}

I.P. Lima*, K. Saito*, D.E. Hamassaki and C.Y.I. Yan

\author{
Departamento de Biologia Celular e do Desenvolvimento, \\ Instituto de Ciências Biomédicas, Universidade de São Paulo, \\ São Paulo, SP, Brasil
}

\author{
Correspondence \\ C.Y.I. Yan \\ Departamento de Biologia Celular \\ e do Desenvolvimento \\ ICB, USP \\ Av. Prof. Lineu Prestes, 1524 \\ Sala 407 \\ 05508-900 São Paulo, SP \\ Brasil \\ Fax: +55-11-3091-7402 \\ E-mail: ireneyan@usp.br \\ *These authors contributed \\ equally to this study. \\ Research supported by FAPESP \\ (No. 01/09047-2). I.P. Lima was \\ the recipient of a CAPES graduate \\ research fellowship. \\ $\ldots \ldots \ldots \ldots \ldots \ldots . . . . .$.
}

Received November 28, 2006 Accepted August 2, 2007 ................................

\begin{abstract}
The retina is a highly differentiated tissue with a complex layered structure that has been extensively characterized. However, most of the previous studies focused on the histology of the central retina while little is known about the cellular composition, organization and function of the marginal retina. Recent research has identified a subpopulation of multipotential progenitor cells in the marginal regions of the retina, closest to the ciliary body ("ciliary marginal zone"). These cells are capable of differentiation in response to an appropriate stimulus. Thus, it is possible that the structure and composition of the marginal retina are distinct from those of the central retina to accommodate the potential addition of newly formed neurons. To characterize the cellular profile of the chick marginal retina, we labeled it immunohistochemically for markers whose staining pattern is well established in the central retina: calbindin, calretinin, protein kinase $\mathrm{C}$, and choline acetyltransferase. Calbindin was present at very low levels in the marginal retina putative photoreceptor layer. Calretinin-positive horizontal cells were also sparse close to the ciliary marginal zone. The bipolar cells in the marginal outer plexiform layer were positive for anti-protein kinase $\mathrm{C}$ antibodies, but the density of labeling was also decreased in relation to the central retina. In contrast, the marginal starburst cholinergic amacrine cell pattern was very similar to the central retina. From these data we conclude that the structure of the marginal retina is significantly different from that of the central retina. In particular, the expression of late retina markers in the marginal retina decreased in comparison to the central retina.
\end{abstract}

Key words - Marginal retina - Ciliary marginal zone - Calbindin - Calretinin ....................

\section{Introduction}

The vertebrate retina plays an important sensory role, conveying visual input to higher levels of the nervous system. Its highly differentiated multilayered structure reflects the physiological complexity required for such a role. The fulfillment of this sensory function takes place mainly in the central region. Accordingly, this region of the retina has been extensively characterized, both in terms of cellular distribution and of the expression of critical markers. In contrast, the cellular distribution and laminar organization of the marginal retina are still poorly understood. The marginal retina is situated next to the 
lens and ciliary body and it differs from the central retina in various functional and morphological aspects, such as visual field, cellular subtype distribution and density (1-3). Finally, the differentiation of the retina during oculogenesis occurs in a central-peripheral wave, so that the marginal retina matures later during development $(4,5)$. While in the central retina cells undergo mitotic arrest and differentiation early during development (E5 in chicks) (6), the marginal retina, which is located $100 \mu \mathrm{m}$ from the edge of the retina, contains proliferating cells even in the adult animal. The mitotic activity of these cells was indirectly identified by their ability to incorporate the thymidine analog bromodeoxyuridine (BrdU) during DNA replication. This proliferating region has been named ciliary marginal zone (CMZ) $(7,8)$.

In chicks, specifically, it has been shown that the proliferating cells of the CMZ can generate amacrine and ganglion cells in the absence of exogenous stimulation (7). Furthermore, upon exogenous application of insulin and FGF2, they can also express markers for amacrine, horizontal, photoreceptor, and ganglion cells $(8,9)$. Lastly, these progenitor cells increase their proliferation upon acute retinal damage (9). Taken together, these data suggest that, similar to lower vertebrates, the progenitor cells of the chicken CMZ are multipotent under adequate conditions and potentially participate in retinal regeneration.

In light of this possibility, further identification of the cellular components of the marginal region and their stratification would aid our understanding of the mechanisms involved in the response of the marginal retina to retinal damage. The accommodation of newly differentiated CMZ cells into the existing marginal network suggests a more plastic histological organization of this region, possibly distinct from the central retina and which might illustrate the sequence of events necessary for retinal regeneration. Moreover, knowledge of the histo- logical organization of the marginal retina could facilitate the identification of the newly differentiated cellular subtypes through their laminar localization, as is done in the central retina.

In the present study, we histologically characterized the marginal retina, including the CMZ, using key markers for central retina cell types (photoreceptors and horizontal, bipolar, amacrine, and ganglion cells): calbindin, calretinin, protein kinase C (PKC), and choline acetyltransferase enzyme (ChAT). The patterns for these markers were compared with previously characterized central patterns to establish, through their expression, the equivalent marginal cell layers.

\section{Material and Methods}

\section{Animals}

One-day-old (P1) Leghorn chicks (Gallus gallus, obtained from Kunitomo Chicken Farm, Mogi das Cruzes, SP, Brazil) were maintained on a 12-h light/dark cycle until P5, when they were sacrificed around 10:00 am with an overdose of ketamine ( $30 \mathrm{mg} / 100 \mathrm{~g}$ body weight) and xylazine ( $6 \mathrm{mg} / 100 \mathrm{~g}$ body weight), and their eyes were rapidly removed. After cutting away the anterior pole or the posterior poles for central or marginal retina experiments, the vitreous humor was removed and the eyes were immersed in ice-cold 2-4\% paraformaldehyde in $0.1 \mathrm{M}$ phosphate buffer (PB) at $\mathrm{pH}$ 7.4 for $3 \mathrm{~h}$. The tissue was then cryoprotected in $30 \%(\mathrm{w} / \mathrm{v})$ sucrose for at least $48 \mathrm{~h}$, frozen in embedding medium (O.C.T., Sakura Finetek, Torrance, CA, USA), cut perpendicularly to the vitreal on a cryostat $(12-\mu \mathrm{m}$ transverse sections), and collected on gelatin-coated glass slides.

These experiments were approved by the Ethics Committee for Animal Research of the Biomedical Sciences Institute, University of São Paulo (protocol number 052), and were conducted in accordance with NIH guidelines. 


\section{Bromodeoxyuridine detection}

Chicks were anesthetized with ketamine (5 mg/100 g body weight) and xylazine ( $1 \mathrm{mg} /$ $100 \mathrm{~g}$ body weight) and $20 \mu \mathrm{L} \mathrm{BrdU}(10 \mu \mathrm{g} ; 5-$ bromo-2-deoxyuridine; Sigma, St. Louis, MO, USA) was injected in the right eye at P1, P2, and P4. The contralateral control eye (left) was injected with $20 \mu \mathrm{L}$ saline. These chicks were sacrificed at P5 and processed as mentioned above. Retinal sections were incubated in $2 \mathrm{M} \mathrm{HCl}$ for $60 \mathrm{~min}$ at $37^{\circ} \mathrm{C}$ for DNA denaturation, washed three times in $\mathrm{PB}$, and blocked with $3 \%$ normal goat serum, $1 \%$ bovine serum albumin and $0.5 \%$ Triton $\mathrm{X}-100$ in PB for $30 \mathrm{~min}$. They were then incubated overnight with rat anti-BrdU primary antibody (Accurate Chemicals, 1:200, Westbury, NY, USA) in $0.5 \%$ Triton $\mathrm{X}-100$ and $3 \%$ normal goat serum in $0.1 \mathrm{M}$ PB. After extensive washes in $0.1 \mathrm{M} \mathrm{PB}$, sections were incubated with anti-rat Alexa-488 secondary antibody (1:1000, Molecular Probes, Eugene, OR, USA). After several washes in PB, slides were coverslipped using Vecta Shield (Vector Laboratories Inc., Burlingame, CA, USA). The results were analyzed with a Nikon PCM2000 (Melville, NY, USA) confocal microscope. The control sections without the primary antibody were not labeled (data not shown).

\section{Immunohistochemistry}

The following primary antibodies were used: anti-calretinin (1:500, Chemicon $\mathrm{AB}$ 1550, Temecula, CA, USA) $(10,11)$, antiChAT (1:100, Chemicon AB144P) (11), anticalbindin (1:200, Sigma C8666) (1) and antiPKC- $\alpha(1: 1000$, Sigma P4334) $(12,13)$. Retinal sections were incubated with the primary antibodies overnight and with Alexa-488 or TRITC-conjugated secondary antibodies (1:1000 or 1:200, respectively; Molecular Probes) for $2 \mathrm{~h}$. Propidium iodide labeling was performed after the secondary antibody washes. The slides were treated with RNAse A (10 mg/mL; Sigma) for $30 \mathrm{~min}$ and incu- bated with $20 \mathrm{mg} / \mathrm{mL}$ propidium iodide (Sigma) for $30 \mathrm{~s}$. The slides were then washed and coverslipped with Vectashield and analyzed with a Nikon PCM2000 confocal microscope.

In addition to anti-calbindin, fluorescently labeled peanut agglutinin (PNA; Vector Laboratories, FL-1071 at 1:50 dilution) was also used as a cone-specific marker $(14,15)$.

\section{Results}

The marginal retina encompasses the CMZ, which contains a population of proliferating progenitor cells. To identify these proliferating cells in the marginal retina and to determine the size of the CMZ, we detected the incorporation of the thymidine analog BrdU. BrdU was injected intraocularly and its presence detected by immunofluorescence (Figure 1D,E). Confirming previous reports, BrdU-positive cells were concentrated in a region $100 \mu \mathrm{m}$ from the edge of the retina where it meets with the retinal pigmented epithelium (7). There were no BrdU-positive cells in the central retina (data not shown). Thus, for our analysis, we defined the CMZ as the peripheral-most 100$\mu \mathrm{m}$ stretch inside the retina that clearly contains proliferating cells. To compare between the CMZ and its surrounding non-proliferating region, we also analyzed a region extending $250 \mu \mathrm{m}$ into the retina from the interface of the retina and the ora serrata. We refer to this region as the marginal retina, which includes the CMZ. Finally, the central retina was defined as the dorsotemporal area above the pecten.

For an initial comparison of the layers present in central and marginal retinas, we used propidium iodide, a nuclear marker (Figure 1A-C). As expected, in the central retina the nuclear layers alternated with plexiform layers (Figure 1A). In the marginal regions, the ganglion cell, inner nuclear and plexiform layers continued from their respective layers observed in the central retina 

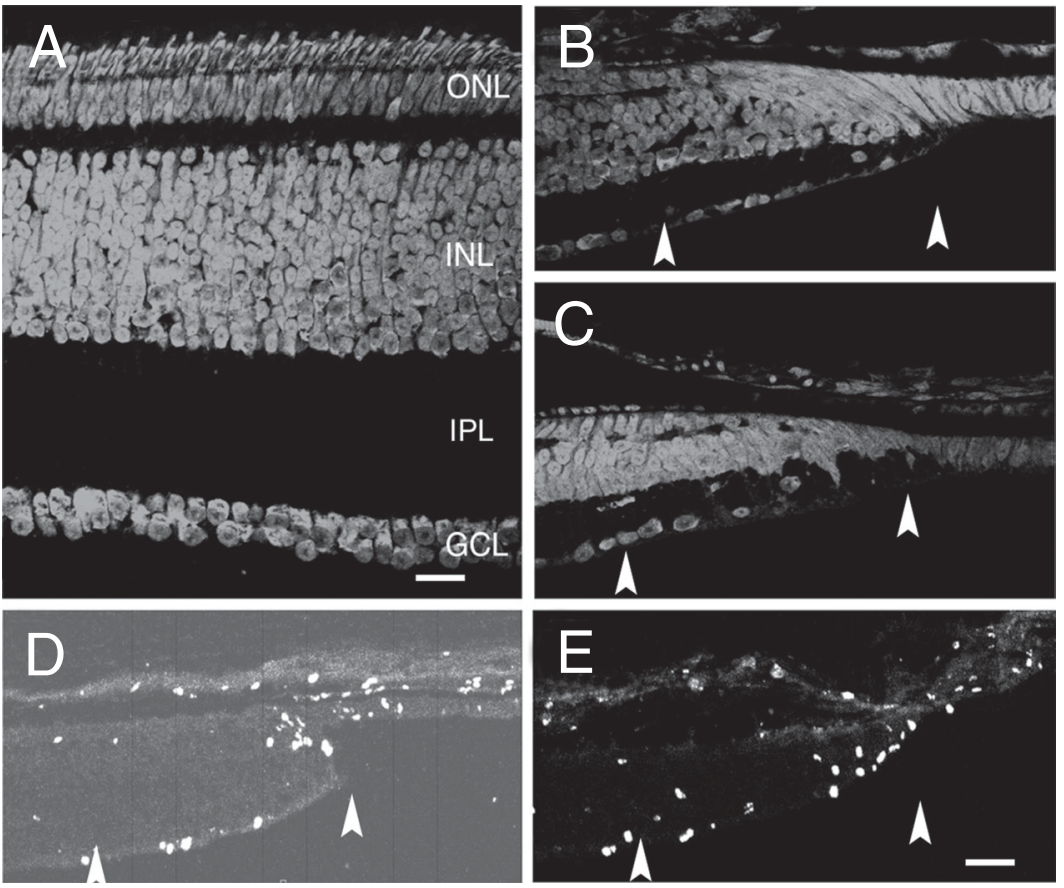

Figure 2. Calbindin-positive cells in central and marginal retina. $A$, Central retina; $B$, temporal marginal retina; $C$, nasal marginal retina. $\mathrm{ONL}=$ outer nuclear layer; INL = inner nuclear layer; $\mathrm{GCL}=$ ganglion cell layer. The vitreous surface faces the bottom of the figure. Arrowheads indicate beginning and end of the ciliary marginal zone. Scale bars in $A=40 \mu \mathrm{m}$ and in $B$ and $C=50$ $\mu \mathrm{m}$.
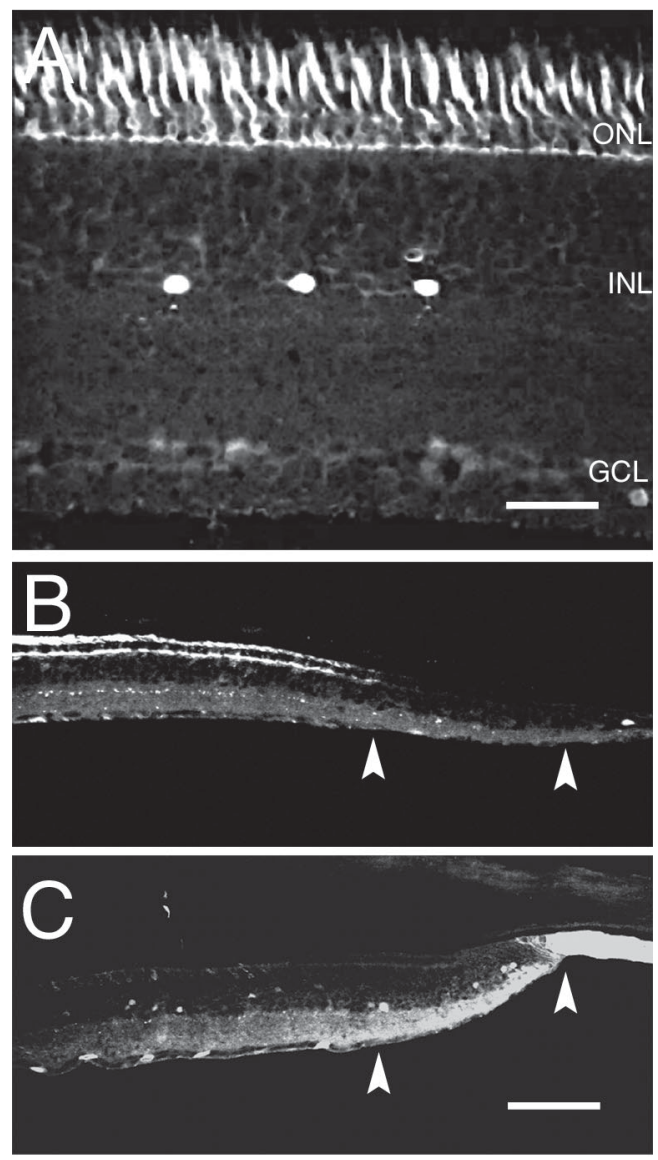

Figure 1. Central and marginal retinal histology. $A-C$, Propidium iodide nuclear staining. $A$, Central retina; $B$, temporal marginal retina; $C$, nasal marginal retina. ONL $=$ outer nuclear layer; $\mathrm{INL}=$ inner nuclear layer; $\mathrm{IPL}=$ inner plexiform layer; $\mathrm{GCL}=$ ganglion cell layer. $D$ and $E$, Proliferating cells in the retinal ciliary marginal zone labeled with bromodeoxyuridine. $D$, Nasal marginal retina; $E$, temporal marginal retina. The vitreous surface faces the bottom of the figure. Arrowheads indicate beginning and end of the ciliary marginal zone. Scale bar $=20 \mu \mathrm{m}$ in all panels.

but, as soon as they approached the CMZ, these layers narrowed (Figure 1B,C). The outer nuclear layer was interrupted and reduced, and the outer plexiform layer practically disappeared at the ciliary-most marginal region. In addition, cells in the outermost layers presented a fusiform shape rather than the more spherical shape observed in the central retina. This initial characterization indicated that the marginal retina is histologically distinct from the central retina, with a decrease in fibers and modifications in cell shape.

To determine whether the relative contribution and distribution of the different cell types in each nuclear layer also change, we analyzed in the marginal retina the expression pattern of different central retinal cell markers that characterize subgroups of retinal cell types.

\section{Calbindin}

Confirming previous studies $(10,16)$, calbindin in the central retina was predominantly found in photoreceptors, and in the inner nuclear (bipolar and amacrine cells) and ganglion cell layers (Figure 2A). In the marginal retina furthest from the $\mathrm{CMZ}$, the distribution of calbindin-positive cells in the putative marginal inner nuclear layer was similar to that of the central retina (Figure $2 \mathrm{~A}-\mathrm{C})$. However, labeling at the ganglion 
cell layer of the CMZ was increased in intensity (Figure 2C). In contrast, when approaching the CMZ, the intensity of calbindin expression in the outer nuclear layer decreased significantly (Figure 2B and C). The nasal and temporal retinas differed in the extent of decrease. Specifically, calbindin immunoreactivity extended further into the ciliary border in the nasal retina than in the temporal retina: while in the nasal retina the absence of calbindin-positive cells occurred near the $\mathrm{CMZ}$, in the temporal marginal retina, the labeling for photoreceptors decreased much earlier (compare Figure 2B and C).

To further analyze the differences between central and marginal photoreceptors, we also used the lectin PNA, which labels cone-associated matrix, primarily the outer and inner segments of the cone (17). Similar to the calbindin-labeling pattern in the marginal retina, the binding of PNA in this region was very weak (data not shown).

\section{Protein kinase C- $\alpha$}

In the central retina, PKC has been located in bipolar cell bodies and their dendrites and axons going to the outer and inner plexiform layers (IPL), respectively (Figure $3 \mathrm{~A})$. In the IPL, the immunolabeling appeared in a punctiform pattern (Figure 3B,C).

The labeling of the inner nuclear layer extended until the CMZ both in the temporal and nasal marginal retina (Figure 3B,C). The main differences in PKC immunoreactivity were found in the outer plexiform layer, which became faint and discontinuous in both the nasal and temporal CMZ. In addition, in the inner nuclear layer of the marginal regions furthest from the $\mathrm{CMZ}$, PKC was detected both in cell bodies and bipolar projections. The PKC-containing cell bodies were mostly concentrated in the innermost portion of the inner nuclear layer, whereas in the central retina, they were located preferentially in the external portions.

\section{Calretinin}

Besides labeling in horizontal cells, calretinin labeling was observed in amacrine and ganglion cells of the central retina, as previously described (Figure 4) (7,10). Although we detected calretinin immunoreactivity in the putative marginal amacrine cells, labeling of horizontal cells decreased significantly when approaching the CMZ. A decrease in labeling was also seen in their processes in the outer plexiform layer.

An additional difference between central and marginal retinas was a decrease in labeling in the IPL. Whereas the central IPL was strongly labeled, labeling in the marginal retina IPL was weak and punctate (compare Figure 4A, B and C). In the CMZ even this sparse labeling in the IPL disappeared. In the

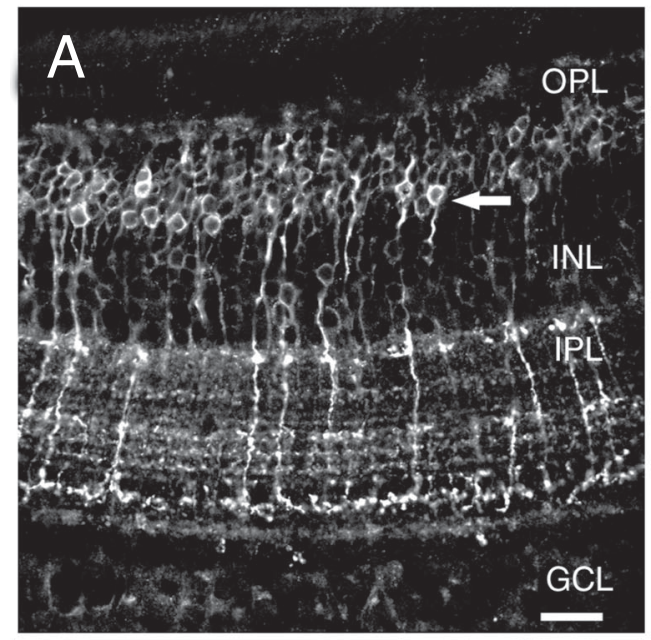

Figure 3. Identification of protein kinase $\mathrm{C}$ - $\alpha$-positive bipolar cells. $A$, Central retina. The arrow points to a labeled central bipolar cell; $B$, temporal marginal retina. The arrow points to a labeled marginal cell that emits processes towards the plexiform layer; $C$, nasal marginal retina. $\mathrm{OPL}=$ outer plexiform layer; INL = inner nuclear layer; IPL = inner plexiform layer; $\mathrm{GCL}=$ ganglion cell layer. The vitreous surface faces the bottom of the figure. Arrowheads indicate beginning and end of the ciliary marginal zone. Scale bars in $A=20$ $\mu \mathrm{m}$ and in $B$ and $C=50 \mu \mathrm{m}$.
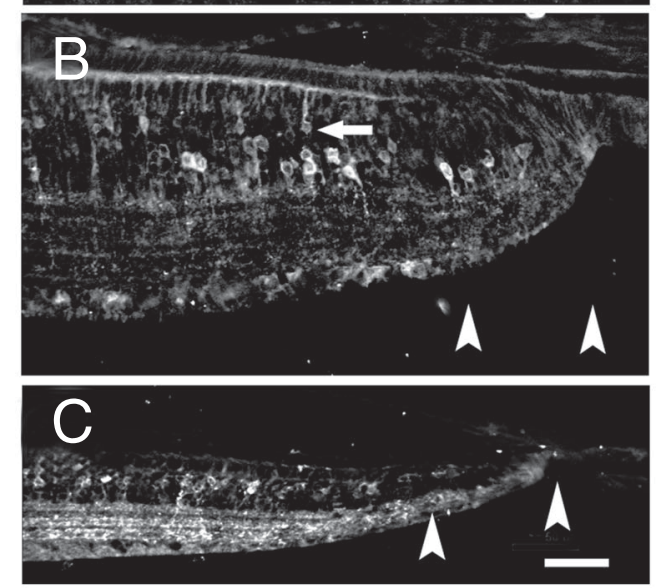
Figure 4. Comparison of calretinin-positive cells in central and marginal retina. $A$, Central retina. The arrow points to a labeled central horizontal cell; $B$, temporal marginal retina; $C$, nasal marginal retina. $\mathrm{OPL}=$ outer plexiform layer; $I N L=$ inner nuclear layer; $\mathrm{GCL}=$ ganglion cell layer. The vitreous surface faces the bottom of the figure. Arrowheads indicate beginning and end of the ciliary marginal zone. Scale bar $=20 \mu \mathrm{m}$ in all panels.

Figure 5. Choline acetyltransferase-immunopositive cells. $A$ Central retina; $B$, temporal marginal retina; $C$, nasal marginal retina. INL = inner nuclear layer; $\mathrm{GCL}=$ ganglion cell layer. The vitreous surface faces the bottom of the figure. Arrowheads indicate beginning and end of the ciliary marginal zone. Scale bars in $A=10 \mu \mathrm{m}$ and in $B$ and $C=15$ $\mu \mathrm{m}$.
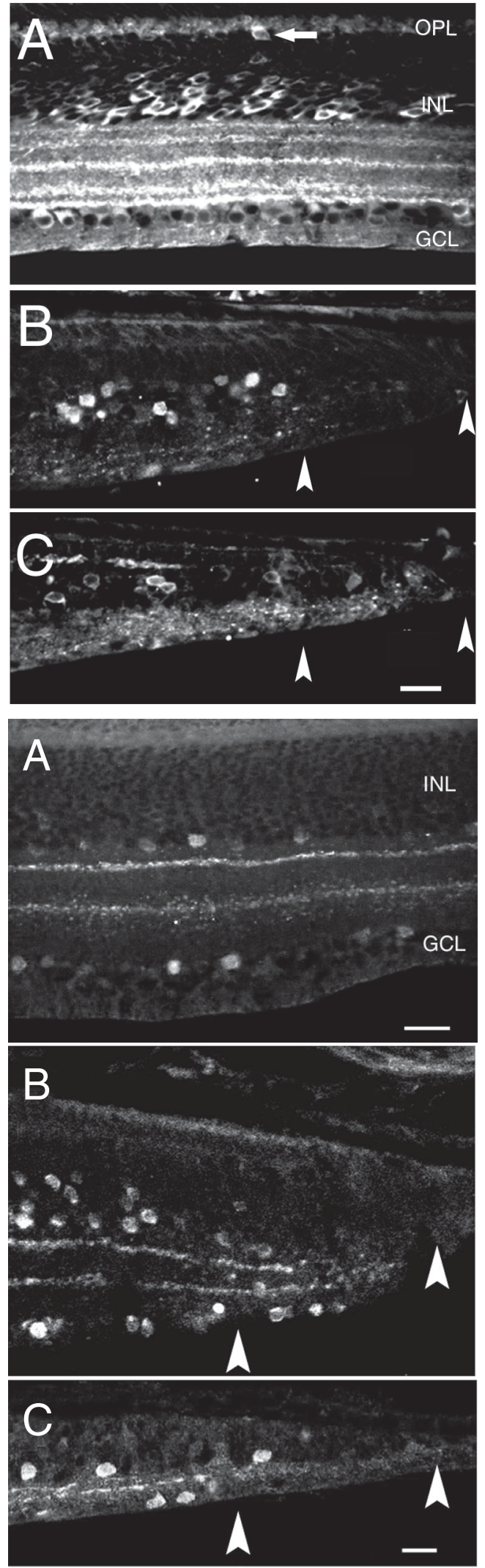

ganglion cell layer a similar but less pronounced decrease in calretinin staining was observed towards the CMZ (Figure 4B,C).

\section{Choline acetyltransferase}

Confirming the results of Spira and collaborators (18), ChAT was detected in amacrine cells located in the inner nuclear layer and in the ganglion cell layer of the central retina, branching into two bands of the IPL (Figure 5A).

In the marginal retina closest to the central region, ChAT-immunolabeling was found in cells with morphology similar to that of the central amacrine cells, and in processes in the IPL (Figure 5A-C). In the CMZ, however, the two ChAT-positive bands were no longer parallel but converged, and amacrine cells of the inner nuclear layer and ganglion cell layer in the $\mathrm{CMZ}$ approached each other as well (Figure 5B,C). This convergence most likely is due to a gradual reduction of the layers that results in the thinning of the retina as it nears the ora serrata.

\section{Discussion}

Previous studies have extensively analyzed the histology of the central retina, demonstrating that this structure is divided into distinct layers composed of cell bodies and processes. Similarly, the marginal retina also displays distinct layers, each with a characteristic cellular distribution. However, our data show that in the marginal retina some layers are modified relative to the central retina. The differences between the central and marginal retina are summarized in Table 1 and were mainly found in the outermost layers. For example, in the marginal retina there was a reduction in the labeling for calbindin and PNA in photoreceptor layers. Moreover, the PKC immunoreactivity of bipolar cell dendrites in the outer plexiform layer and calretinin-positive horizontal cells disappears in the marginal zone. 
Calbindin is mainly associated with central cone photoreceptors and amacrine cells in diurnal animals (19-21). Here, we show that calbindin-labeling was absent in the putative photoreceptor layer of the chick marginal retina. An explanation would be that the cones in the marginal zone might not be sufficiently differentiated to express calbindin. Calbindin is first seen in photoreceptors of the central retina in E16 chick embryos (16). Moreover, previous studies have shown that the temporal CMZ contains more proliferating progenitor cells than the nasal CMZ (7), which means that the temporal marginal zone potentially contains more undifferentiated cells. Thus, if calbindin is a marker of the final stages of photoreceptor differentiation, the calbindin-negative marginal photoreceptor layer should be more extensive in the temporal retina than in the nasal retina. Accordingly, we observed that the temporal marginal photoreceptor layer lost calbindin-labeling much farther from the CMZ than the nasal marginal retina. Therefore, one explanation for the calbindinnegative marginal photoreceptor layer could be that this region is populated with photoreceptors that are not yet terminally differentiated. Retardation in marginal photoreceptor differentiation could also explain the decrease in PNA binding in the putative marginal photoreceptor layer. Other lines of evidence support this possibility. For instance, photoreceptor oil droplets appear first in the center and later in the periphery (22), and the developmental timing and distribution of wavelength-specific cone opsin expression also differs from center to periphery (1).

An alternative explanation is that the marginal retina - especially closer to the $\mathrm{CMZ}$ - is maintained in an undifferentiated state. This possibility is supported by a detailed analysis of the CMZ of older postnatal chicks (7). Neither calbindin nor visinin was detected in the outer nuclear layer of this region even in P14 animals. In contrast, cell proliferation in the $\mathrm{CMZ}$ is maintained at least up to P19, suggesting that the adult $\mathrm{CMZ}$ is optimized for the maintenance of undifferentiated proliferating cells and therefore does not support differentiation. In this scenario, daughter cells of the CMZ progenitor pool would have to leave the CMZ environment to express markers of a more differentiated state. This hypothesis is supported by cell fate experiments. Proliferating cells from the CMZ migrate away from the margin and are sometimes found in the granular cell layer and outer nuclear layer of the non-proliferating marginal retina $(8,23)$. Cell-type specific markers begin to appear when the cells are farther from the CMZ $(7,24)$.

Finally, another explanation for the lack of calcium-binding proteins at the periphery would be that marginal cones have lower intracellular calcium levels. The immunoreactivity of calbindin, calretinin and parvalbumin depends on the occupancy of their calcium-binding sites. These proteins are better recognized by their respective antibodies in the presence of calcium (25). Thus,

\begin{tabular}{|c|c|c|c|c|}
\hline & Calbindin & Calretinin & ChAT & PKC \\
\hline \multicolumn{5}{|l|}{ ONL } \\
\hline Central & + & - & - & - \\
\hline Marginal & - & - & - & - \\
\hline \multicolumn{5}{|l|}{ OPL } \\
\hline Central & - & - & - & ++ \\
\hline Marginal & - & - & - & + \\
\hline \multicolumn{5}{|l|}{ INL } \\
\hline Central & +++ & +++ & ++ & +++ \\
\hline Marginal & +++ & ++ & ++ & +++ \\
\hline \multicolumn{5}{|l|}{ IPL } \\
\hline Central & ++ & ++ & ++ & +++ \\
\hline Marginal & ++ & ++ & ++ & +++ \\
\hline \multicolumn{5}{|l|}{$\mathrm{GCL}$} \\
\hline Central & ++ & ++ & ++ & + \\
\hline Marginal & ++ & ++ & ++ & + \\
\hline
\end{tabular}

The intensity of calbindin, calretinin, choline acetyl transferase (ChAT), and protein kinase $C(P K C)$ labeling was assessed qualitatively for the outer nuclear layer (ONL), outer plexiform layer (OPL), inner nuclear layer (INL), inner plexiform layer (IPL), and ganglion cell layer (GCL). + indicates intensity of labeling; - indicates absence of labeling. 
the decrease in marginal labeling for calbindin could be caused by diminished intracellular calcium levels and not by a decrease in calbindin itself.

Differences in the histology and immunolabeling pattern of the marginal photoreceptor layer, irrespective of the cause, suggest that additional marginal retinal components could also be distinct from those in the central retina. This difference should be reflected in the pattern of immunolabeling for other retina cell type markers associated with the inner layers. To assess this point, we employed antibodies against PKC- $\alpha$, calretinin, and ChAT, which are respectively markers for bipolar, horizontal, and amacrine cells.

Particularly striking was the disappearance of PKC and calretinin immunoreactivity in the outer plexiform layer. Our data showed that the PKC-positive cells near the CMZ extended neurites towards the IPL but not towards the outer plexiform layer. In contrast, still in the marginal retina, but further from the CMZ, the PKC-positive cells could be seen extending their processes bidirectionally. This pattern is similar to that seen for early bipolar cell development in chick and rat retina: PKC-positive bipolar cells extend neurites first towards the IPL and then establish connections in the outer plexiform layer $(26,27)$. Thus, if we consider the dynamics of PKC immunoreactivity in bipolar cell neurites as an indicator of the state of differentiation of the bipolar cell, the data presented here suggest that they not only are reduced in number in the marginal region, but are also relatively undifferentiated.

Our results with labeling for calretinin also suggest that marginal horizontal cells differ from central ones. Calretinin expression in chick central horizontal cells is only detected from embryonic stage 18 (E18) onwards (16). In the marginal region, calretinin-positive cells in the outermost region of the inner nuclear layer were absent in the neighborhood of the CMZ, suggesting that putative horizontal cells of this region are not sufficiently differentiated to express calretinin. Conversely, calbindin expression in chick central amacrine cells is present by E10 in the innermost part of the inner nuclear layer (16). In our experiments calbindin labeling was detected throughout the corresponding region in the marginal retina. Taken together, these data suggest that the putative inner nuclear layer of the $\mathrm{CMZ}$ expresses markers that are present early in the central retina such as calbindin, but not late markers such as calretinin.

The hypothesis that the marginal zone contains a histologically distinct subpopulation of cells that are still undergoing differentiation is supported by observations in fish and frog retina. Both are capable of retinal neurogenesis in the marginal zone throughout their lives, and their marginal cells display a periphery-central-directed gradient of gene expression that correlates with the differentiation status, with more marginal cells expressing markers of earlier states of differentiation $(23,28,29)$. An equivalent proliferative zone was not found in mammalian CMZ (30). This absence could be due to an evolutionary decrease of the Shh pathway activity in the retina (31). Instead, a proliferative population was found in the mammalian pigmented ciliary margin $(32,33)$. Similar to amphibian and avian CMZ cells, these mammalian adult retinal progenitor cells can also express different retinal markers after arresting from the cell cycle, rendering them a highly promising tool for retinal regenerative therapies.

For targeted regenerations, it would be convenient to control the rate of retinal progenitor proliferation and to direct their differentiation pathway. In chicks, intraocular injections of insulin and fibroblast growth factor stimulate proliferation at the $\mathrm{CMZ}$ (7). This same treatment induces differentiation of progenitors but mostly into amacrine and ganglion cells (9). Conversely, neural 
progenitor cultures derived from mouse ciliary body can be induced in vitro to express a larger variety of cell markers, including photoreceptors and bipolar and Müller glia markers (32,33). This difference in potentiality between in vivo and in vitro settings suggest that the retinal marginal microenvironment might contain elements that restrict the multipotentiality of the adult progenitor cells. Thus, although significant progress has been made in our understanding of vertebrate adult retina progenitors, we believe that further insight into the composition of this region will contribute to our understanding of the fate of the multipotential progenitor cells, as well as the connective plasticity required for incorporating them into the existing neural network.

\section{Acknowledgments}

The authors would like to thank Andréa S. Santos Bredariol for excellent technical advice, and Dora Ventura and Daniela Bonci (Department of Experimental Psychology of the Universidade de São Paulo, SP, Brazil) for sharing the PKC antibody.

\section{References}

1. Bruhn SL, Cepko CL. Development of the pattern of photoreceptors in the chick retina. J Neurosci 1996; 16: 1430-1439.

2. Chen $Y$, Naito J. Regional specialization of ganglion cell layer of the chick retina. J Vet Med Sci 2000; 62: 53-57.

3. Curcio CA, Allen KA. Topography of ganglion cells in human retina. J Comp Neurol 1990; 300: 5-25.

4. Kahn AJ. An autoradiographic analysis of the time of appearance of neurons in the developing chick neural retina. Dev Biol 1974; 38: 3040.

5. McCabe KL, Gunther EC, Reh TA. The development of the pattern of retinal ganglion cells in the chick retina: mechanisms that control differentiation. Development 1999; 126: 5713-5724.

6. Mey J, Thanos S. Development of the visual system of the chick. I. Cell differentiation and histogenesis. Brain Res Brain Res Rev 2000; 32: 343-379.

7. Fischer AJ, Reh TA. Identification of a proliferating marginal zone of retinal progenitors in postnatal chickens. Dev Biol 2000; 220: 197210.

8. Fischer AJ, Reh TA. Growth factors induce neurogenesis in the ciliary body. Dev Biol 2003; 259: 225-240.

9. Fischer AJ, Dierks BD, Reh TA. Exogenous growth factors induce the production of ganglion cells at the retinal margin. Development 2002; 129: 2283-2291.

10. Araki CM, Pires RS, Britto LR, Lindstrom JM, Karten HJ, HamassakiBritto DE. Differential co-localization of nicotinic acetylcholine receptor subunits with calcium-binding proteins in retinal ganglion cells. Brain Res 1997; 774: 250-255.

11. Araki CM, Hamassaki-Britto DE. Calretinin co-localizes with the NMDA receptor subunit NR1 in cholinergic amacrine cells of the rat retina. Brain Res 2000; 869: 220-224.

12. Bonci DM, Lima SM, Grotzner SR, Ribeiro CA, Hamassaki DE, Ventura DF. Losses of immunoreactive parvalbumin amacrine and immunoreactive alphaprotein kinase $\mathrm{C}$ bipolar cells caused by methylmercury chloride intoxication in the retina of the tropical fish Hoplias malabaricus. Braz J Med Biol Res 2006; 39: 405-410.

13. Hamassaki-Britto DE, Brzozowska-Prechtl A, Karten HJ, Lindstrom
JM. Bipolar cells of the chick retina containing alpha-bungarotoxinsensitive nicotinic acetylcholine receptors. Vis Neurosci 1994; 11: 63-70.

14. Blanks JC, Johnson LV. Selective lectin binding of the developing mouse retina. J Comp Neurol 1983; 221: 31-41.

15. Belmonte MA, Santos MF, Kihara AH, Yan CY, Hamassaki DE. Light-induced photoreceptor degeneration in the mouse involves activation of the small GTPase Rac1. Invest Ophthalmol Vis Sci 2006; 47: 1193-1200.

16. Ellis $\mathrm{JH}$, Richards $\mathrm{DE}$, Rogers $\mathrm{JH}$. Calretinin and calbindin in the retina of the developing chick. Cell Tissue Res 1991; 264: 197-208.

17. Blanks JC, Johnson LV. Specific binding of peanut lectin to a class of retinal photoreceptor cells. A species comparison. Invest Ophthalmol Vis Sci 1984; 25: 546-557.

18. Spira AW, Millar TJ, Ishimoto I, Epstein ML, Johnson CD, Dahl JL, et al. Localization of choline acetyltransferase-like immunoreactivity in the embryonic chick retina. J Comp Neurol 1987; 260: 526-538.

19. Chiquet C, Dkhissi-Benyahya O, Chounlamountri N, Szel A, Degrip WJ, Cooper HM. Characterization of calbindin-positive cones in primates. Neuroscience 2002; 115: 1323-1333.

20. Hendrickson A, Djajadi HR, Nakamura L, Possin DE, Sajuthi D. Nocturnal tarsier retina has both short and long/medium-wavelength cones in an unusual topography. J Comp Neurol 2000; 424: 718730.

21. Tolosa de TN, Perez A, Riis R, Smith C, Norman ML, Wasserman $\mathrm{RH}$. Comparative immunolocalization of the plasma membrane calcium pump and calbindin D28K in chicken retina during embryonic development. Eur J Histochem 2002; 46: 333-340.

22. Lopez R, Lopez-Gallardo M, Busturia I, Anezary L, Prada C. Spatial and temporal patterns of growth and differentiation of cone oil droplets in the chick retina. J Neurosci Res 2005; 79: 401-411.

23. Otteson DC, D'Costa AR, Hitchcock PF. Putative stem cells and the lineage of rod photoreceptors in the mature retina of the goldfish. Dev Biol 2001; 232: 62-76.

24. Ghai K, Stanke JJ, Fischer AJ. Patterning of the circumferential marginal zone of progenitors in the chicken retina. Brain Res 2007 
(ahead of print).

25. Winsky L, Kuznicki J. Antibody recognition of calcium-binding proteins depends on their calcium-binding status. J Neurochem 1996; 66: 764-771.

26. Caminos E, Velasco A, Jarrin M, Aijon J, Lara JM. Protein kinase Clike immunoreactive cells in embryo and adult chicken retinas. Brain Res Dev Brain Res 1999; 118: 227-230.

27. Johansson K, Bruun A, deVente J, Ehinger B. Immunohistochemical analysis of the developing inner plexiform layer in postnatal rat retina. Invest Ophthalmol Vis Sci 2000; 41: 305-313.

28. Otteson DC, Hitchcock PF. Stem cells in the teleost retina: persistent neurogenesis and injury-induced regeneration. Vision Res 2003; 43: 927-936.

29. Perron M, Kanekar S, Vetter ML, Harris WA. The genetic sequence of retinal development in the ciliary margin of the Xenopus eye. Dev Biol 1998; 199: 185-200.

30. Kubota R, Hokoc JN, Moshiri A, McGuire C, Reh TA. A comparative study of neurogenesis in the retinal ciliary marginal zone of homeothermic vertebrates. Brain Res Dev Brain Res 2002; 134: 31-41.

31. Moshiri A, Reh TA. Persistent progenitors at the retinal margin of ptc+/- mice. J Neurosci 2004; 24: 229-237.

32. Tropepe V, Coles BL, Chiasson BJ, Horsford DJ, Elia AJ, McInnes RR, et al. Retinal stem cells in the adult mammalian eye. Science 2000; 287: 2032-2036.

33. Ahmad I, Tang L, Pham H. Identification of neural progenitors in the adult mammalian eye. Biochem Biophys Res Commun 2000; 270: 517-521. 